\title{
Cardiorespiratory fitness is positively associated with a healthy dietary pattern in New Zealand adolescents
}

\author{
Anna S Howe ${ }^{1}$, Paula ML Skidmore ${ }^{1}$, Winsome R Parnell ${ }^{1}$, Jyh Eiin Wong ${ }^{1,2}$, \\ Alexandra C Lubransky ${ }^{2}$ and Katherine E Black ${ }^{2, *}$ \\ 'Department of Human Nutrition, University of Otago, Dunedin, New Zealand: ${ }^{2}$ School of Healthcare Sciences, \\ Faculty of Health Sciences, Universiti Kebangsaan Malaysia, 50300 Kuala Lumpur, Malaysia
}

Submitted 11 January 2015: Final revision received 11 June 2015: Accepted 4 August 2015: First published online 8 September 2015

\begin{abstract}
Objective: To examine the association between cardiorespiratory fitness and dietary patterns in adolescents.

Design: Food choice was assessed using the validated New Zealand Adolescent FFQ. Principal components analysis was used to determine dietary patterns. Trained research assistants measured participants' height and body mass. Cardiorespiratory fitness was assessed in a subset of participants using the multistage $20 \mathrm{~m}$ shuttle run. The level and stage were recorded, and the corresponding $\mathrm{VO}_{2 \max }$ was calculated. Differences in mean $\mathrm{VO}_{2 \max }$ according to sex and BMI were assessed using $t$ tests, while associations between cardiorespiratory fitness and dietary patterns were examined using linear regression analyses adjusted for age, sex, school attended, socio-economic deprivation and BMI.

Setting: Secondary schools in Otago, New Zealand.

Subjects: Students ( $n$ 279) aged 14-18 years who completed an online lifestyle survey during a class period.

Results: Principal components analysis produced three dietary patterns: 'Treat Foods', 'Fruits and Vegetables' and 'Basic Foods'. The 279 participants who provided questionnaire data and completed cardiorespiratory fitness testing had a mean age of 15.7 (SD 0.9) years. Mean $\mathrm{VO}_{2 \max }$ was 45.8 (sD 6.9) $\mathrm{ml} / \mathrm{kg}$ per min. The 'Fruits and Vegetables' pattern was positively associated with $\mathrm{VO}_{2 \max }$ in the total sample $(\beta=0.04 ; 95 \%$ CI $0.02,0.07)$, girls $(\beta=0.06 ; 95 \%$ CI $0.03,0 \cdot 10)$ and boys $(\beta=0.03 ; 95 \%$ CI $0 \cdot 01,0 \cdot 05)$.

Conclusions: These results indicate that increase in cardiorespiratory fitness was associated with a healthier dietary pattern, suggesting both should be targeted as part of a global lifestyle approach. Longitudinal studies are needed to confirm this association in relation to health outcomes in New Zealand adolescents.
\end{abstract}

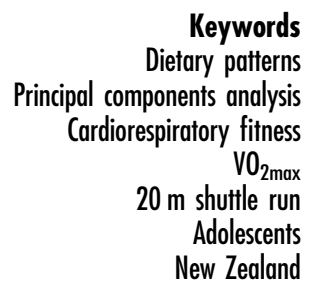

Cardiorespiratory fitness has important health implications during adolescence and throughout adulthood ${ }^{(1)}$. Low levels of cardiorespiratory fitness have been linked to excessive body fatness ${ }^{(2-4)}$, elevations in CVD risk markers including total cholesterol, systolic blood pressure and $\mathrm{TAG}^{(2,3,5)}$, and diabetes risk ${ }^{(2,6,7)}$. In addition, one study examining longitudinal evidence suggests that cardiorespiratory fitness between 13 and 16 years is related to healthier CVD risk profiles (total cholesterol and total cholesterol:HDL cholesterol) 15 years later ${ }^{(5)}$.

FITNESSGRAM $^{\circledR}$ established the Healthy Fitness Zones, using age- and sex-specific cut-offs, to represent the lowest levels of cardiorespiratory fitness linked to adequate health-related outcomes ${ }^{(8)}$. High cardiorespiratory fitness, defined as meeting or exceeding the FITNESSGRAM ${ }^{\circledR}$ standard (criterion $\mathrm{VO}_{2 \max }$ of $42 \mathrm{ml} / \mathrm{kg}$ per min and $35 \mathrm{ml} / \mathrm{kg}$ per $\min$ for 14-18-year-old boys and girls, respectively ${ }^{(8)}$ ), has been found to be positively associated with healthier cardiovascular risk profiles, healthier body compositions and improved insulin sensitivity ${ }^{(9)}$.

Nutrition is another important modifiable risk factor associated with health. Diets habitually containing high intakes of fruits and vegetables can confer many health benefits including lower risk of developing certain cancers and prevention of obesity and $\mathrm{CVD}^{(10)}$. As both cardiorespiratory fitness and nutrition may be important to adolescents' current and future health it is important to investigate whether they are associated, as these may be 
potential targets for interventions in order to prevent undesirable behaviours impacting adolescent health or becoming lifelong habits.

The few studies investigating cardiorespiratory fitness and nutrition have mostly focused on specific food groups or nutrient intakes, mostly in adults ${ }^{(11-16)}$; of the studies in adolescents ${ }^{(17-19)}$, the results have not always been congruent. Cardiorespiratory fitness has been found to be positively associated with higher usual intakes of fruit, vegetables, bread and dairy products ${ }^{(16,20)}$, and negatively associated with sweetened beverages in both adolescents ${ }^{(17)}$ and young adults (aged 23-27 years) ${ }^{(13)}$. These results are reflected in studies examining usual nutrient intakes, with high cardiorespiratory fitness being associated with higher intakes of folate, vitamins $\mathrm{A}$ and $\mathrm{C}, \mathrm{Ca}$ and fibre, and lower intakes of fat and sucrose ${ }^{(13)}$. However, despite these positive findings others have found no evidence of an association $^{(18,19)}$.

As the current research suggests inconsistent agreement between cardiorespiratory fitness and food group and nutrient intakes, further research is required in order to clarify associations. Dietary patterns may offer a novel way to investigate dietary intake by taking into account the way foods are consumed in combination. So far only one study has investigated dietary patterns and cardiorespiratory fitness, and this was in adults ${ }^{(16)}$. The authors found that increasing 'meat' pattern score was negatively associated with treadmill duration (in seconds) in 'white' males $(\beta=-36 \cdot 71$ (SE 8.24), $P<0.0001)$ and 'white' females $(\beta=18.82$ (sE 9.04), $P<0.05$ ) but not the 'black' males or females. The reverse was true for the 'fruit-vegetable' pattern, so that increase in this pattern score was positively associated with treadmill duration (in seconds) but in 'white' males only ( $\beta=11.65$ (SE 5.45), $P<0.05)^{(16)}$. These results are not dissimilar to the themes seen in previous work investigating food group and nutrient intakes $^{(13,17)}$ and suggest that cardiorespiratory fitness and a healthy diet may be part of an overall healthy lifestyle. However, more studies are required to confirm these associations, particularly among adolescents.

Therefore, the aim of the present study was to investigate the association between cardiorespiratory fitness and dietary patterns in adolescents. It was hypothesised that cardiorespiratory fitness would be positively associated with dietary patterns reflecting high fruit and vegetable consumption, and negatively associated with dietary patterns reflecting greater consumption of 'treat' type foods.

\section{Methods}

\section{Study design and participants}

The present study analysed data collected from 279 school students as part of the Otago School Students Lifestyle Survey Two (OSSLS2), a cross-sectional study conducted from February to June 2011 in the Otago province of New Zealand, which collected information from
730 participants. Data were collected on food choice, psychosocial correlates of diet, eating behaviours and body composition. Secondary schools in the Otago area were invited to take part in the study. Randomly selected classes from school years 11 to 13 in each school were invited to take part, with one class from each school year in smaller schools and up to four classes in larger schools invited. All testing took place during school hours, with the exact time depending on the schools' timetable and as previously described $^{(21)}$. In the week before the projected data collection, invited students were given information sheets and consent forms for themselves and their parents. Students were required to sign a consent form in order to participate, while parents were only required to sign a form if they wished their child to opt out of the study. The recruitment of OSSLS2 participants has been reported in further detail elsewhere ${ }^{(21)}$.

\section{Survey}

The survey was administered online during class time in the schools' computer rooms. Students supplied information on their date of birth, age, sex, ethnicity and residential address. Ethnicity was categorised into three groups: Māori, Pacific or 'New Zealand European and Other'. School decile was determined by the deprivation level, as measured by census data ${ }^{(22)}$, of students attending the school, with the lowest decile rating reflecting the $10 \%$ of schools nationwide with students mostly from high deprivation areas and the highest decile rating reflecting the upper $10 \%$ of schools with students from mostly low deprivation areas $^{(23)}$. School decile was divided into 'middle' (deciles 5 to 8) and 'high' (deciles 9 and 10).

Food consumption was assessed using the New Zealand Adolescent FFQ adapted from the Health Behaviour in School-aged Children study ${ }^{(24)}$. This questionnaire was pre-tested in a sample of adolescents from Otago before use in OSSLS2 and showed good repeatability and relative validity $^{(25)}$. Participants were asked: 'On average, how many times a week do you usually eat or drink any of the following foods?' The food items assessed were 'fruit', 'vegetables', 'sweets' (e.g. jelly beans, marshmallows, etc.), 'chocolate confectionery' (e.g. chocolate, Moro, Crunchie, etc.), 'standard milk' ( $\approx 3.3 \%$ total fat), 'other milk' (low-fat, rice, soya), 'cheese', 'breakfast cereals', 'white bread', 'brown/ wholegrain bread', 'potato crisps', 'hot chips/fries', 'artificially sweetened soft drinks' (e.g. Diet Coke, Coke Zero, etc.), 'regular soft drinks' (e.g. Coke, Pepsi, etc.), 'energy drinks' (e.g. Red Bull), 'sports drinks' (e.g. Powerade), 'fruit juice/ fruit drinks' and 'alcohol'. Regular soft drinks, energy drinks and sports drinks were collapsed into one food group called 'sugar-sweetened soft drinks'.

\section{Body composition}

Height and weight were collected during the time the students completed the online survey. Students had their 
height measured twice to the nearest millimetre using a calibrated portable stadiometer (manufactured by the Physical Education Department Workshops for use in the Life in New Zealand survey, University of Otago) and weight, in light clothing, measured to the nearest kilogram using a calibrated segmental bioelectrical impedance analyser (BC-418; Tanita Corporation, Japan), by trained research assistants. BMI was calculated and thinness, normal-weight, overweight and obese categories were determined using the 2012 International Obesity Task Force cut-off points ${ }^{(26)}$. Because the prevalence of obese individuals in the current sample was small, the overweight and obese groups were combined, hereafter referred to as 'overweight'. Likewise, those with a low BMI-for-age (thinness) were combined with the normal-weight, hereafter referred to as 'non-overweight'.

\section{Cardiorespiratory fitness testing}

Fitness testing was undertaken on a convenience sub-sample of the participants from the OSSLS2, as participants had to opt in to the fitness testing. Léger et al.'s ${ }^{(27)} 20 \mathrm{~m}$ multistage fitness test was used to determine cardiorespiratory fitness. All study personnel were trained prior to data collection. The $20 \mathrm{~m}$ multistage fitness test was performed on a tennis court or in a gymnasium, at the participants' schools. Participants were instructed to run between two parallel lines of cones, $20 \mathrm{~m}$ apart, pacing themselves in accordance with the pre-recorded beeps emitted from a stereo system. The test began with a pace of $8.5 \mathrm{~km} / \mathrm{h}$, increasing by $0.5 \mathrm{~km} / \mathrm{h}$ every minute (or stage). If a participant failed to reach the line of cones before the next beep sounded they were warned. If two consecutive beeps were missed the participant was withdrawn from the test and their last stage and lap were recorded.

The $20 \mathrm{~m}$ multistage fitness test results were used to determine $\mathrm{VO}_{2 \max }$ relative to body mass ( $\mathrm{ml} / \mathrm{kg}$ per $\mathrm{min}$ ) for each participant using the predictive equations of Léger et $a l^{(27)}$, which have been previously validated in adolescents $^{(27)}$. Participants were additionally categorised into low and high cardiorespiratory fitness using the FITNESSGRAM $^{\circledR}$ standards for the Healthy Fitness Zone cut-offs (criterion $\mathrm{VO}_{2 \max }$ of $42 \mathrm{ml} / \mathrm{kg}$ per min and $35 \mathrm{ml} / \mathrm{kg}$ per min for 14-18-year-old boys and girls, respectively) ${ }^{(8)}$.

\section{Statistical analysis}

Statistical analyses were undertaken using the statistical software package Stata version 12.0IC. Dietary pattern scores were derived using principal components analysis in the entire OSSLS2 sample. The principal components analysis was based on sixteen food items (fruit, vegetables, sweets, chocolate confectionery, standard milk, other milks, cheese, breakfast cereals, white bread, brown/wholegrain bread, potato crisps, hot chips/fries, artificially sweetened soft drinks, sugar-sweetened soft drinks, fruit juice and alcohol).
Principal components analysis produced three components, which explained $46.7 \%$ of the total variance within the food data: (i) a 'Treat Foods' pattern (sweets, chocolate confectionery, potato crisps, sugar-sweetened and artificially sweetened soft drinks); (ii) a 'Fruits and Vegetables' pattern (fruit, vegetables, cheese and brown/multigrain bread); and (iii) a 'Basic Foods' pattern (standard milk, other milks, white bread and breakfast cereals). The development of these patterns has been reported in further detail elsewhere ${ }^{(21)}$.

Only participants who had complete data for all variables were included in the final analysis ( $n$ 279). Differences in sample characteristics were examined in two ways: (i) using $\chi^{2}$ tests, accounting for clusters, for categorical variables presented as unadjusted numbers and percentages; and (ii) continuous variables were presented as means and standard deviations adjusted for clustering, and differences were assessed with linear regression with sandwich estimators to adjust the standard errors and school as the clustering unit.

Dietary patterns derived from principal components analysis were examined as the dependent variables, while cardiorespiratory fitness (continuous $\mathrm{VO}_{2 \max }$ and FITNESSGRAM $^{\circledR}$ categories) was the independent variable in regression modelling. Linear regression analysis was undertaken using the regress command in Stata. Sandwich estimators were used to adjust the standard errors with the robust command in Stata and school as the clustering unit because schools were the primary sampling unit. Model assumptions were checked ${ }^{(28)}$. In total four models were used to examine these associations. Model 1 was adjusted for age. As sex ${ }^{(29)}$, socio-economic status (school decile $)^{(30)}$ and $\mathrm{BMI}^{(21)}$ have been found to be associated with food choice, these factors were included in model 2. As boys and girls have been shown to have differing associations with dietary patterns ${ }^{(21)}$, model 3 and model 4 were run separately for boys and girls, with model 3 adjusted for age and model 4 adjusted for age, BMI category and school decile. Cardiorespiratory fitness $\times$ sex interactions were examined.

A two-sided $P$ value of $<0.05$ was considered statistically significant.

\section{Results}

\section{Demographic characteristics}

Of those who took part in the online survey (730 participants), a convenience sub-sample of $40 \%$ also completed the cardiorespiratory fitness testing. For the current analyses 279 participants (38\% of all survey participants) provided complete demographic, food and fitness data (thirteen participants who completed the fitness testing did not have sufficient online survey data to be included in the current analysis). Of the eleven schools surveyed in the OSSLS2 study, only one school had no 
survey participants complete the cardiorespiratory fitness testing. However, students from the missing school represented only $1.5 \%$ of those who completed the study (data not shown). Students who took part in the cardiorespiratory testing did not differ from those who did not by sex, ethnicity, school year, BMI or pattern scores. However, a higher proportion of students who took part in the cardiorespiratory fitness tests were from a 'high decile' school (65\%) compared with those who did not take part in the testing ( $57 \% ; P=0.048)$.

The mean age of participants was 15.7 (SD 0.4) years and ranged from 14 to 18 years. Most of the students included in the current analyses were 'New Zealand European and Other' (90\%) and just over half of the students were in school year 11 (Table 1). The highest proportion of students was from the least deprived neighbourhoods (data not shown), in agreement with $65 \%$ of students attending a 'high decile' school (Table 1). Most students were non-overweight (78\%; Table 1), and of the 279 participants $6 \%$ were classified as having a low BMI-for-age and 5\% were obese. Girls who completed the cardiorespiratory fitness testing had significantly lower 'Treat Foods' and 'Basic Foods' scores and higher 'Fruits and Vegetables' scores than the boys $(P<0.05$; Table 1). Higher levels of cardiorespiratory fitness were seen in boys compared with girls and in those who were non-overweight compared with those classified as overweight (Table 2).

\section{Continuous $\mathrm{VO}_{2 \max }$ models}

The association between $\mathrm{VO}_{2 \max }$ and the 'Treat Foods' dietary pattern for boys was a negative linear association ( $\beta=-0.024 ; 95 \%$ CI $-0.037,-0 \cdot 011$; Table 3 ). The association between $\mathrm{VO}_{2 \max }$ and the 'Fruits and Vegetables' dietary pattern for the total sample was a positive linear association ( $\beta=0.04 ; 95 \%$ CI 0.013, 0.066; Table 3), indicating that with increasing fitness, adolescents reported consuming more fruit, vegetables, cheese and multigrain bread. A significant $\mathrm{VO}_{2 \max } \times$ sex interaction was seen in the 'Fruits and Vegetables' model only $(P<0 \cdot 05)$.

\section{FITNESSGRAM ${ }^{\circledR}$ models}

Despite the potential loss of information ${ }^{(31)}$, categorisation can be justified if a cut-off point is related to health, as is the case with the FITNESSGRAM ${ }^{\circledR}$ cut-offs. With this in mind, and in order to allow for comparison between other studies, linear regression analyses were also run using the FITNESSGRAM $^{\circledR}$ cut-offs (Table 4). Those classified by the FITNESSGRAM $^{\circledR}$ cut-offs as having high cardiorespiratory fitness had lower 'Treat Foods' pattern scores than individuals classified as having low cardiorespiratory fitness $(\beta=-0.56$; $95 \% \mathrm{CI}-1 \cdot 05,-0 \cdot 06$; Table 4$)$.

Table 1 Sample characteristics of fitness participants in the OSSLS2, stratified by sex $(n$ 279)

\begin{tabular}{|c|c|c|c|c|c|c|c|}
\hline & \multicolumn{2}{|c|}{ Total sample ( $n$ 279) } & \multicolumn{2}{|c|}{ Girls (n 146) } & \multicolumn{2}{|c|}{ Boys ( $n$ 133) } & \multirow[b]{2}{*}{$P+$} \\
\hline & $n$ & $\% *$ & $n$ & $\%^{*}$ & $n$ & $\%^{*}$ & \\
\hline \multicolumn{8}{|l|}{ Sex } \\
\hline Girls & 146 & 52 & - & - & - & - & - \\
\hline Boys & 133 & 48 & - & - & - & - & \\
\hline \multicolumn{8}{|l|}{ Ethnicity } \\
\hline Māori & 22 & 8 & 14 & 10 & 8 & 6 & \\
\hline Pacific & 7 & 2 & 4 & 2 & 3 & 2 & \\
\hline NZEO & 250 & 90 & 128 & 88 & 122 & 92 & 0.528 \\
\hline \multicolumn{8}{|l|}{ School year } \\
\hline 11 & 148 & 53 & 91 & 62 & 57 & 43 & \\
\hline 12 & 66 & 24 & 29 & 20 & 37 & 28 & \\
\hline 13 & 65 & 23 & 26 & 18 & 39 & 29 & 0.551 \\
\hline \multicolumn{8}{|l|}{ School decile } \\
\hline Middle & 99 & 35 & 39 & 27 & 60 & 45 & \\
\hline High & 180 & 65 & 107 & 73 & 73 & 55 & 0.001 \\
\hline \multicolumn{8}{|l|}{ BMI } \\
\hline Non-overweight & 219 & 78 & 112 & 77 & 107 & 80 & \\
\hline \multirow[t]{2}{*}{ Overweight } & 60 & 22 & 34 & 23 & 26 & 20 & 0.676 \\
\hline & Mean & SD & Mean & SD & Mean & SD & $P \S$ \\
\hline \multicolumn{8}{|l|}{ Pattern scores $\ddagger$} \\
\hline Treat Foods & -0.04 & 0.30 & -0.29 & 0.39 & 0.24 & 0.18 & 0.011 \\
\hline Fruits and Vegetables & 0.11 & 0.22 & 0.28 & 0.41 & -0.08 & 0.12 & 0.005 \\
\hline Basic Foods & 0.02 & 0.36 & -0.13 & 0.33 & 0.18 & 0.40 & 0.025 \\
\hline
\end{tabular}

OSSLS2, Otago School Students Lifestyle Survey Two; NZEO, New Zealand European and Other; CRF, cardiorespiratory fitness; PCA, principal components analysis.

${ }^{\star}$ Column percentage, unadjusted for confounders.

$\dagger P$ of $x^{2}$ test for sex differences in CRF participants, adjusted for clustering by school.

$\ddagger$ Presented as means and standard deviations adjusted for clustering by school.

$\S P$ of linear regression with the PCA-derived dietary patterns as the dependent variables, sex as the independent variable, additionally adjusted for age, overweight status and school decile, with clustering by school and robust standard errors. 
Table 2 CRF variables for fitness participants in the OSSLS2, stratified by sex and BMI classification

\begin{tabular}{|c|c|c|c|c|c|c|c|c|c|}
\hline & \multicolumn{3}{|c|}{$20 \mathrm{~m}$ shuttle run (stage) } & \multicolumn{3}{|c|}{$\mathrm{VO}_{2 \max }(\mathrm{ml} / \mathrm{kg}$ per min $)$} & \multicolumn{3}{|c|}{ High CRF according to the FITNESSGRAM ${ }^{\circledR}$ cut-offs* } \\
\hline & Mean & sD $†$ & $P \ddagger$ & Mean & sD $†$ & $P \ddagger$ & $n$ & $\% \S$ & $P \|$ \\
\hline Total sample ( $n$ 279) & $7 \cdot 3$ & 1.5 & - & $45 \cdot 8$ & 3.8 & - & 246 & 88 & - \\
\hline \multicolumn{10}{|l|}{ Sex } \\
\hline Girls (n 146) & $6 \cdot 3$ & $1 \cdot 2$ & & $43 \cdot 3$ & 3.3 & & 133 & 90 & \\
\hline Boys ( $n$ 133) & 8.3 & 1.2 & $<0.001$ & 48.5 & 3.2 & $<0.001$ & 113 & 85 & 0.453 \\
\hline \multicolumn{10}{|l|}{ BMI category } \\
\hline Non-overweight ( $n$ 219) & 7.6 & 1.3 & & $46 \cdot 8$ & 3.4 & & 207 & 95 & \\
\hline Overweight $(n 60)$ & 5.9 & 1.8 & 0.003 & $42 \cdot 2$ & 4.9 & 0.003 & 39 & 65 & 0.003 \\
\hline
\end{tabular}

CRF, cardiorespiratory fitness; OSSLS2, Otago School Students Lifestyle Survey Two; PCA, principal components analysis.

${ }^{*}$ Criterion $\mathrm{VO}_{2 \max }$ of $42 \mathrm{ml} / \mathrm{kg} / \mathrm{min}$ and $35 \mathrm{ml} / \mathrm{kg} / \mathrm{min}$ for $14-18$-year-old boys and girls, respectively ${ }^{(7)}$.

†Standard deviations adjusted for clustering by school.

fLinear regression with the PCA-derived dietary patterns as the dependent variables, sex or BMI category as the independent variable, additionally adjusted for age, sex or overweight status, and school decile, with clustering by school and robust standard errors.

$\S$ Row percentage, unadjusted for confounders.

$\| P$ for $X^{2}$ test for differences between sexes and BMI categories, adjusted for clustering by school.

Table 3 Linear associations between CRF (continuous $\mathrm{VO}_{2 \max }$ ) and PCA-derived dietary patterns in Otago adolescents $(n$ 279)

\begin{tabular}{|c|c|c|c|c|c|c|c|c|c|}
\hline \multirow[b]{2}{*}{$\mathrm{VO}_{2 \max }$} & \multicolumn{3}{|c|}{ Treat Foods } & \multicolumn{3}{|c|}{ Fruits and Vegetables } & \multicolumn{3}{|c|}{ Basic Foods } \\
\hline & $\beta^{*}$ & $95 \% \mathrm{Cl}$ & $P$ & $\beta^{\star}$ & $95 \% \mathrm{Cl}$ & $P$ & $\beta^{*}$ & $95 \% \mathrm{Cl}$ & $P$ \\
\hline \multicolumn{10}{|c|}{ Total sample ( $n$ 279) } \\
\hline Model 1 & 0.001 & $-0.019,0.020$ & 0.938 & 0.024 & $-0.001,0.049$ & 0.060 & 0.001 & $-0.021,0.022$ & 0.961 \\
\hline Model 2 & -0.014 & $-0.034,0.006$ & 0.156 & $0.040 \dagger$ & $0.013,0.066$ & 0.008 & -0.007 & $-0.032,0.017$ & 0.510 \\
\hline \multicolumn{10}{|c|}{ Girls only ( $n 146)$} \\
\hline Model 3 & -0.013 & $-0.056,0.031$ & 0.510 & 0.061 & $0.025,0.097$ & 0.005 & -0.022 & $-0.076,0.032$ & 0.367 \\
\hline Model 4 & -0.004 & $-0.038,0.028$ & 0.750 & 0.064 & $0.030,0.098$ & 0.003 & -0.014 & $-0.058,0.030$ & 0.484 \\
\hline \multicolumn{10}{|c|}{ Boys only ( $n$ 133) } \\
\hline Model 3 & -0.019 & $-0.037,-0.001$ & 0.040 & 0.024 & $-0.001,0.049$ & 0.057 & -0.004 & $-0.033,0.025$ & 0.765 \\
\hline Model 4 & -0.024 & $-0.037,-0.011$ & 0.004 & 0.027 & $0.001,0.054$ & 0.047 & -0.004 & $-0.038,0.030$ & 0.771 \\
\hline
\end{tabular}

CRF, cardiorespiratory fitness; PCA, principal components analysis.

Model 1 = adjusted for age; model $2=$ adjusted for age, overweight, school decile and sex; model $3=$ adjusted for age; model $4=$ adjusted for age, overweight and school decile.

*Linear regression with PCA-derived dietary patterns as the dependent variables, $\mathrm{VO}_{2 \max }$ as the independent variable, with clustering by school and robust standard errors. Unstandardised regression coefficient; expected change in the mean dietary pattern score for every unit change in VO ${ }_{2 m a x}$.

†Significant sex $\times \mathrm{VO}_{2 \max }$ interaction observed, $R^{2}$ increased from 0.0985 to $0.1129, P=0.014$.

Table 4 Associations between those defined as having high CRF by the FITNESSGRAM ${ }^{\circledR}$ Healthy Fitness Zone cut-offs ${ }^{\star}$ and PCA-derived dietary patterns, compared with those with low fitness, in Otago adolescents $(n$ 279)

\begin{tabular}{|c|c|c|c|c|c|c|c|c|c|}
\hline \multirow[b]{2}{*}{ High CRF } & \multicolumn{3}{|c|}{ Treat Foods } & \multicolumn{3}{|c|}{ Fruits and Vegetables } & \multicolumn{3}{|c|}{ Basic Foods } \\
\hline & $\beta \dagger$ & $95 \% \mathrm{Cl}$ & $P$ & $\beta \dagger$ & $95 \% \mathrm{Cl}$ & $P$ & $\beta \dagger$ & $95 \% \mathrm{Cl}$ & $P$ \\
\hline \multicolumn{10}{|c|}{ Total sample (n 246/279)‡ } \\
\hline Model 1 & -0.56 & $-1.05,-0.06$ & 0.031 & 0.30 & $-0.03,0.63$ & 0.073 & -0.36 & $-0.74,0.01$ & 0.054 \\
\hline Model 2 & -0.53 & $-0.96,-0.09$ & 0.023 & 0.22 & $-0.07,0.51$ & 0.118 & -0.29 & $-0.58,-0.01$ & 0.045 \\
\hline \multicolumn{10}{|c|}{ Girls only $(n 133 / 146) \ddagger$} \\
\hline Model 3 & -0.78 & $-1.53,-0.03$ & 0.044 & 0.28 & $-0.33,0.89$ & 0.316 & -0.62 & $-1 \cdot 34,0.10$ & 0.082 \\
\hline Model 4 & -0.73 & $-1.47,0.01$ & 0.054 & 0.17 & $-0.68,1.01$ & 0.652 & -0.47 & $-1.06,0.11$ & 0.099 \\
\hline \multicolumn{10}{|c|}{ Boys only $(n 113 / 133) \ddagger$} \\
\hline Model 3 & -0.31 & $-0.72,0.14$ & 0.145 & 0.25 & $0.05,0.45$ & 0.022 & $-0 \cdot 12$ & $-0.30,0.07$ & 0.165 \\
\hline Model 4 & -0.48 & $-0.87,-0.10$ & 0.022 & 0.30 & $-0.03,0.64$ & 0.071 & $-0 \cdot 18$ & $-0.40,0.04$ & $0 \cdot 100$ \\
\hline
\end{tabular}

CRF, cardiorespiratory fitness; PCA, principal components analysis.

Model 1 =adjusted for age; model 2 =adjusted for age, sex, overweight and school decile; model $3=$ adjusted for age; model $4=$ adjusted for age, overweight and school decile.

${ }^{*}$ Criterion $\mathrm{VO}_{2 \max }$ of $42 \mathrm{ml} / \mathrm{kg}$ per $\mathrm{min}$ and $35 \mathrm{ml} / \mathrm{kg}$ per min for 14-18-year-old boys and girls, respectively ${ }^{(7)}$.

†Linear regression with PCA-derived dietary patterns as the dependent variables, FITNESSGRAM ${ }^{\circledR}$ category as the independent variable, with clustering by school and robust standard errors. Unstandardised regression coefficient; expected change in the mean dietary pattern score for those defined as having high CRF compared with those with low CRF.

$\ddagger$ Number of those defined as having high CRF/total number of CRF participants. 


\section{Discussion}

To our knowledge, the present study is the first to use dietary pattern analysis to examine the association between cardiorespiratory fitness and overall diet in adolescents and the most recent study to measure cardiorespiratory fitness in older New Zealand adolescents. Dietary patterns were examined as the outcome variable in order to determine how diet differs between cardiorespiratory fitness levels of adolescents. The study suggests fitter adolescents have healthier dietary patterns. Fitter individuals had higher 'Fruits and Vegetables' dietary pattern scores and lower 'Treat Foods' dietary pattern scores than less fit adolescents, as indicated by their higher reported frequency of consumption of fruit, vegetables, cheese and brown/wholegrain bread and lower frequency of consumption of sweets, chocolates, crisps, fries and sugar-sweetened drinks.

The limited numbers of studies investigating cardiorespiratory fitness and diet have not always found congruent results. Positive findings were found in a large European study ${ }^{(17)}$ and no evidence of an association was seen in two smaller American studies ${ }^{(18,19)}$. The current study aimed to clarify associations between cardiorespiratory fitness and diet in adolescents with the use of dietary patterns, which may offer a novel way to investigate dietary intake by taking into account the way foods are consumed in combination. This being said, the results of the current study are similar to previous work investigating cardiorespiratory fitness and food groups ${ }^{(17)}$. European adolescents classified as having high fitness were found to have lower mean usual intakes of 'sweetened beverages', a component of the current 'Treat Foods' pattern, than those classified as having low cardiorespiratory fitness ${ }^{(17)}$. Congruent with the findings of previous studies investigating dietary patterns in adults ${ }^{(16)}$, the 'Fruits and Vegetables' pattern score increased with increasing cardiorespiratory fitness, indicating that those with high cardiovascular fitness reported more frequent consumption of fruits and vegetables than less fit individuals. These results are also in agreement with work investigating fitness and food groups ${ }^{(17,19)}$.

As the previous studies have not presented standardised coefficients, it is hard to compare effect sizes. CuencaGarcía et al. ${ }^{(17)}$ reported an unstandardised coefficient for $\mathrm{VO}_{2 \max }(\mathrm{ml} / \mathrm{kg}$ per min) and fruit intake of 1.337 (95\% CI $0 \cdot 168,2 \cdot 506)$ and 1.351 (95\% CI -0.013, 2.715) for boys and girls, respectively, while the current study found much smaller coefficients of 0.06 (95\% CI 0.04, 0.09) and 0.03 (95\% CI 0.00, 0.05). As the current analysis uses dietary patterns, which take into account multiple food items, and the dietary pattern scores have been standardised, the smaller effect sizes seen in the current study are not unexpected and may still have important public health implications. The current results suggest an approximate half-a-standard-deviation difference for 'Treat Foods' score between those with high cardiorespiratory fitness and those with low. Half-a-standard-deviation difference in the 'Treat Foods' pattern translates to approximately half an extra eating occasion of sweets, chocolates, crisps, fries, artificially sweetened soft drinks, and two extra eating occasions of sugar-sweetened soft drinks each week. Unfortunately, as we cannot determine the dietary energy from these extra eating occasions, we cannot determine the clinical significance in terms of potential weight gain. However, we do know that aerobic fitness is inversely associated with adiposity in children ${ }^{(32)}$ and therefore any extra energy intake by the least fit group is probably undesirable from a body composition and health perspective.

The current research suggests that fitter adolescents are consuming healthier diets. The least fit adolescents may consume 'unhealthy' foods with increased frequency and 'healthy' foods with decreased frequency because they lack nutrition knowledge or their consumption pattern may be part of an unhealthy lifestyle. As the current study assessed only frequency of intake we cannot rule out the possibility that less fit individuals consume 'unhealthy' foods in smaller portions frequently while fitter individuals eat larger portions less frequently; and vice versa for the 'healthier' foods. However, previous research results, which adjusted for energy intake, are consistent with the current study ${ }^{(17,19)}$. Although we did control for weight status in our models there was a high proportion of participants in the normal-weight BMI category and this may have influenced the results. Whether such a finding would have been seen in a population with a more diverse BMI cannot be concluded.

To date, the present and previous studies examining cardiorespiratory fitness and diet ${ }^{(17,19)}$ have been crosssectional and as such causality cannot be determined. However, physical activity may help explain this association. It is possible that those with a higher cardiorespiratory fitness are more active and more likely to be health conscious, so consume less treat/snack type foods. While it is outside the scope of the current paper to investigate a possible mechanism of action, recent evidence suggests that physical activity and eating behaviour may be related through a common neurocognitive pathway. A study in women suggested that moderate-to-vigorous activity might reduce neurologically determined food motivation, regardless of BMI classification ${ }^{(33)}$. However, that study had a small sample and assessed only short-term activity and food motivation. Nevertheless, if these results are repeatable, they support the neurocognitive association between activity and eating behaviour suggested by a recent review ${ }^{(34)}$. The review postulated that physical activity might help modify eating behaviour and thus help address the obesity epidemic $^{(34)}$.

The current study found a lack of evidence for an association between $\mathrm{VO}_{2 \max }$ and the 'Basic Foods' pattern. However, previous work investigating cardiorespiratory fitness and intake of food groups suggests that fitter 
individuals had higher intake amounts of bread and dairy products $^{(17)}$. Carbohydrate (e.g. sports drinks) and protein drinks (made with milk) can be common preexercise and exercise recovery foods ${ }^{(35)}$, with one study estimating $17 \%$ of $16-18$-year-old boys using protein supplements ${ }^{(36)}$. Therefore it may be likely that the association between $\mathrm{VO}_{2 \max }$ and these foods is a reflection of this behaviour in fitter individuals. Future research is required to ascertain the nature of the association between $\mathrm{VO}_{2 \max }$ and the 'Basic Foods' pattern, if one does actually exist.

The present study is the most recent one to measure cardiorespiratory fitness in New Zealand adolescents, in an internationally comparable way. While little recent literature exists on cardiorespiratory fitness in New Zealand, the current results are not dissimilar to those of a smaller New Zealand study in slightly younger adolescents $(10-15 \text { years })^{(37)}$. The use of the multistage $20 \mathrm{~m}$ shuttle run to assess cardiorespiratory fitness in the current study allowed direct comparison with other large studies in adolescent populations. In comparison to European adolescents of a similar age, the current sample completed more stages in the test resulting in higher predicted $\mathrm{VO}_{2 \max }$ and a higher prevalence of those classified as having high cardiorespiratory fitness than the European adolescents $^{(38)}$. The comparatively higher levels of cardiorespiratory fitness seen in the current sample are most likely related to the self-selected nature of our fitness sample, which is reflected in the high proportion of individuals being classified as having high cardiorespiratory fitness by the FITNESSGRAM ${ }^{\circledR}$ cut-offs.

There are several limitations to the current study. Comparison of cardiorespiratory fitness is difficult between studies as it can be assessed in various ways with numerous methodologies available within laboratory and field settings. However, the current study used the multistage $20 \mathrm{~m}$ shuttle run to assess cardiorespiratory fitness which, despite relying on predictive equations to estimate $\mathrm{VO}_{2 \max }$, has been found to have reasonable validity and reliability in adolescent populations ${ }^{(27)}$. Further, as participants volunteered to participate in the fitness testing it is possible that those who were fitter were the ones who completed the testing compared with the less fit. However, this is an issue for any study where voluntary consent is required. Despite this the results suggest that there were some participants whose fitness levels were poor, suggesting that it was not only the more fit students who participated, and as previously stated there were no differences in the demographic characteristics of those who completed the fitness testing and those who only completed the online survey. Environmental impacts, in particular heat and humidity, may be another potential limitation with the multistage $20 \mathrm{~m}$ shuttle run ${ }^{(39)}$. In the current study participants were exposed to a wide range of weather conditions as data were collected from late summer to early winter. In order to standardise this, and the running surface, testing was completed inside the schools' own gymnasiums where possible. Although testing was completed throughout the school day, the maximal nature of the testing means that there is unlikely to be any effect from circadian variation in $\mathrm{VO}_{2}$ uptake kinetics ${ }^{(40)}$. Physical activity levels may influence aerobic fitness; however, it has been shown that aerobic fitness is independently associated with adiposity in children ${ }^{(32)}$ and therefore the aim of the present study was to investigate fitness rather than physical activity.

Some students may have terminated the test before true maximal capacity, or exhaustion, was reached. However, this is a problem all studies are likely to incur unless they measure $\mathrm{VO}_{2 \max }$ directly and apply a threshold to ensure maximal capacity is reached ${ }^{(41)}$. Trained research assistants encouraged students to push themselves and it is likely that as the test was completed in groups, students encouraged and competed against each other.

Despite these limitations, the multistage $20 \mathrm{~m}$ shuttle run method is good for use in epidemiological studies as it is inexpensive, large numbers can be tested in a relatively short time frame (e.g. a school class period) and the procedure is easy to implement and follow.

The high proportion of participants of 'New Zealand European and Other' ethnicity and of higher socioeconomic status potentially limits generalisibility to the New Zealand population. An Australian study investigating weight-related behaviours in children found differences in cardiorespiratory fitness according to socio-economic status and ethnicity. Middle-Eastern and Asian children from low-socio-economic homes were more likely to be classified as having low cardiorespiratory fitness by FITNESSGRAM $^{\circledR}$ than their 'English-speaking' counterparts ${ }^{(42)}$. These results suggest that ethnicity may be important in relation to cardiorespiratory fitness, although the authors do not propose reasons for these differences. It is possible that different cultures engage in activity differently, or place a different value on activity during childhood, and this results in differences in fitness levels. Despite the lack of diversity within the current sample, most secondary schools in Otago were sampled. In addition, the current study is the first to complete fitness testing in older New Zealand adolescents and despite the small sample size did find significant associations. Due to the self-selection into the fitness testing by students from the main study the high levels of cardiorespiratory fitness in the current sample are of little surprise and may have resulted in attenuation of the associations and bias estimates due to the under-representation of individuals with low fitness.

The FFQ used in the present study was pilot tested and validated in the sample population ${ }^{(25)}$; however, the FFQ measured only frequency not amount of foods. Therefore we are unable to adjust for energy, as such we can only comment on the frequency of reported consumption. Nevertheless, dietary pattern analysis is a novel way of examining how foods are consumed in combination and 
extends previous work which focused on individual food groups and nutrient intakes. While a commonly cited limitation of dietary patterns is their apparent lack of generalisibiliy, previous studies have found similar types of patterns ${ }^{(43,44)}$.

\section{Conclusion}

In conclusion, the current study adds to the limited literature investigating cardiorespiratory fitness and diet by providing evidence that fitter adolescents have healthier dietary patterns than less fitter adolescents. Longitudinal studies are needed to confirm these associations in relation to health outcomes. Future studies could investigate the differences in eating habits between the fit and unfit individuals and these differences could be targeted as part of an intervention. As we have shown that those with lower cardiorespiratory fitness have less healthy dietary patterns, improvements to both diet and fitness should be targeted as part of a global healthy lifestyle approach.

\section{Acknowledgements}

Acknowledgements: The authors would like to thank all participating schools for providing computer facilities and access to students during their class time, and assisting with supervision of students during data collection. They also thank all students who participated in the study and all those who were involved in data collection and processing. The authors also thank Associate Professor Sheila Williams from the Department of Preventive and Social Medicine for her invaluable statistical advice. Financial support: This study was supported by grants from the University of Otago, Lottery Health Research of New Zealand, and the National Heart Foundation of New Zealand. The funders had no role in the design, analysis or writing of this article. Conflict of interest: None. Authorship: A.S.H., P.M.L.S. and K.E.B. were responsible for conception of this particular study and performing data analyses. A.S.H. drafted the initial manuscript. P.M.L.S. and K.E.B. are the principal investigators for the overall OSSLS2 project, were responsible for conception and design of the project, and oversaw questionnaire design, data collection and processing. A.S.H., J.E.W. and A.C.L. contributed to the design of the project, including questionnaire design and data collection and processing. W.R.P. also contributed to study design. All authors provided critical review and revision of the manuscript and have read and approved the final version of the manuscript. Ethics of buman subject participation: This study was conducted according to the guidelines laid down in the Declaration of Helsinki and all procedures involving human subjects were approved by the University of Otago Human Ethics Committee. Written informed consent was obtained from all participants.

\section{References}

1. Eisenmann JC, Wickel EE, Welk GJ et al. (2005) Relationship between adolescent fitness and fatness and cardiovascular disease risk factors in adulthood: the Aerobics Center Longitudinal Study (ACLS). Am Heart J 149, 46-53.

2. Hurtig-Wennlöf A, Ruiz JR, Harro M et al. (2007) Cardiorespiratory fitness relates more strongly than physical activity to cardiovascular disease risk factors in healthy children and adolescents: the European Youth Heart Study. Eur J Cardiovasc Prev Rehabil 14, 575-581.

3. Janz KF, Dawson JD \& Mahoney LT (2002) Increases in physical fitness during childhood improve cardiovascular health during adolescence: the Muscatine Study. Int J Sports Med 23, Suppl. 1, S15-S21.

4. Moliner-Urdiales D, Ruiz JR, Vicente-Rodriguez G et al. (2011) Associations of muscular and cardiorespiratory fitness with total and central body fat in adolescents: the HELENA study. Br J Sports Med 45, 101-108.

5. Twisk JWR, Kemper HCG \& van Mechelen W (2002) The relationship between physical fitness and physical activity during adolescence and cardiovascular disease risk factors at adult age. The Amsterdam Growth and Health Longitudinal Study. Int J Sports Med 23, Suppl. 1, S8-S14.

6. Ruiz JR, Rizzo NS, Ortega FB et al. (2007) Markers of insulin resistance are associated with fatness and fitness in school-aged children: the European Youth Heart Study. Diabetologia 50, 1401-1408.

7. Dwyer T, Magnussen CG, Schmidt MD et al. (2009) Decline in physical fitness from childhood to adulthood associated with increased obesity and insulin resistance in adults. Diabetes Care 32, 683-687.

8. Cureton KJ \& Warren GL (1990) Criterion-referenced standards for youth health-related fitness tests: a tutorial. Res $Q$ Exerc Sport 61, 7-19.

9. Lobelo F, Pate RR, Dowda M et al. (2009) Validity of cardiorespiratory fitness criterion-referenced standards for adolescents. Med Sci Sports Exerc 41, 1222-1229.

10. World Health Organization (2003) Diet, Nutrition and the Prevention of Chronic Diseases. Report of a Joint WHO/FAO Expert Consultation. WHO Technical Report Series no. 916. Geneva: WHO.

11. Blair SN, Ellsworth NM, Haskell WL et al. (1981) Comparison of nutrient intake in middle-aged men and women runners and controls. Med Sci Sports Exerc 13, 310-315.

12. Butterworth DE, Nieman DC, Underwood BC et al. (1994) The relationship between cardiorespiratory fitness, physical activity, and dietary quality. Int J Sport Nutr 4, 289-298.

13. Haraldsdóttir J \& Andersen LB (1994) Dietary factors related to fitness in young men and women. Prev Med 23, 490-497.

14. Brodney S, McPherson RS, Carpenter RA et al. (2001) Nutrient intake of physically fit and unfit men and women. Med Sci Sports Exerc 33, 459-467.

15. Finley CE, LaMonte MJ, Waslien CI et al. (2006) Cardiorespiratory fitness, macronutrient intake, and the metabolic syndrome: the Aerobics Center Longitudinal Study. J Am Diet Assoc 106, 673-679.

16. Shikany JM, Jacobs DR, Lewis CE et al. (2013) Associations between food groups, dietary patterns, and cardiorespiratory fitness in the Coronary Artery Risk Development in Young Adults study. Am J Clin Nutr 98, 1402-14069.

17. Cuenca-García M, Ortega FB, Huybrechts I et al. (2012) Cardiorespiratory fitness and dietary intake in European adolescents: the Healthy Lifestyle in Europe by Nutrition in Adolescence study. Br J Nutr 107, 1850-1859.

18. Gray A \& Smith C (2003) Fitness, dietary intake, and body mass index in urban Native American youth. J Am Diet Assoc 103, 1187-1191.

19. Lloyd T, Chinchilli V, Rollings N et al. (1998) Fruit consumption, fitness, and cardiovascular health in female adolescents: the 
Penn State Young Women's Health Study. Am J Clin Nutr 67, 624-630.

20. Moreira C, Santos R, Moreira P et al. (2013) Cardiorespiratory fitness is negatively associated with metabolic risk factors independently of the adherence to a healthy dietary pattern. Nutr Metab Cardiovasc Dis 23, 670-676.

21. Howe AS, Black KE, Wong JE et al. (2013) Dieting status influences associations between dietary patterns and body composition in adolescents: a cross-sectional study. Nutr J 12, 51.

22. Salmond C, Crampton P \& Atkinson J (2007) NZDep2006 Index of Deprivation User's Manual. Wellington: Department of Public Health, University of Otago.

23. Ministry of Education (2012) New Zealand Schools. Education Counts 2012. Wellington: Ministry of Education.

24. Vereecken C \& Maes L (2003) A Belgian study on the reliability and relative validity of the Health Behaviour in School-Aged Children food-frequency questionnaire. Public Health Nutr 6, 581-588.

25. Wong JE, Parnell WR, Black KE et al. (2012) Reliability and relative validity of a food frequency questionnaire to assess food group intakes in New Zealand adolescents. Nutr J 11, 65 .

26. Cole TJ \& Lobstein T (2012) Extended international (IOTF) body mass index cut-offs for thinness, overweight and obesity. Pediatr Obes 7, 284-294.

27. Léger LA, Mercier D, Gadoury C et al. (1988) The multistage 20 metre shuttle run test for aerobic fitness. J Sports Sci 6, 93-101.

28. Osborne J \& Waters E (2002) Four assumptions of multiple regression that researchers should always test. Pract Assess Res Eval 8, 1-5.

29. Scully M, Dixon H, White V et al. (2007) Dietary, physical activity and sedentary behaviour among Australian secondary students in 2005. Health Promot Int 22, 236-245.

30. Cutler GJ, Flood A, Hannan P et al. (2011) Multiple sociodemographic and socioenvironmental characteristics are correlated with major patterns of dietary intake in adolescents. J Am Diet Assoc 111, 230-240.

31. Altman DG \& Royston P (2006) The cost of dichotomising continuous variables. BMJ 332, 1080.

32. Johnson MS, Figueroa-Colon R, Herd SL et al. (2000) Aerobic fitness, not energy expenditure, influences subsequent increase in adiposity in black and white children. Pediatrics 106, E50.

33. Hanlon B, Larson MJ, Bailey BW et al. (2012) Neural response to pictures of food after exercise in normal-weight and obese women. Med Sci Sports Exerc 44, 1864-1870.

34. Joseph RJ, Alonso-Alonso M, Bond DS et al. (2011) The neurocognitive connection between physical activity and eating behaviour. Obes Res 12, 800-812.

35. Ivy JL \& Ferguson-Stegall LM (2013) Nutrient timing: the means to improved exercise performance, recovery, and training adaptation. Am J Lifestyle Med 8, 2460259.

36. Mattila VM, Parkkari J, Laakso L et al. (2010) Use of dietary supplements and anabolic-androgenic steroids among Finnish adolescents in 1991-2005. Eur J Public Health 20, 306-311.

37. Benson AC, Torode ME \& Singh MAF (2006) Muscular strength and cardiorespiratory fitness is associated with higher insulin sensitivity in children and adolescents. Int J Pediatr Obes 1, 222-231.

38. Ortega FB, Artero EG, Ruiz JR et al. (2011) Physical fitness levels among European adolescents: the HELENA study. Br J Sports Med 45, 20-29.

39. Olds T, Tomkinson G, Léger L et al. (2006) Worldwide variation in the performance of children and adolescents: an analysis of 109 studies of the 20-m shuttle run test in 37 countries. J Sports Sci 24, 1025-1038.

40. Reilly T \& Brooks GA (1986) Exercise and the circadian variation in body temperature measures. Int J Sports Med $\mathbf{7}$, $358-362$

41. Armstrong N \& Welsman JR (1994) Assessment and interpretation of aerobic fitness in children and adolescents. Exerc Sport Sci Rev 22, 435-476.

42. Hardy LL, Lesley K, Hector D et al. (2013) Socio-cultural differences in Australian primary school children's weight and weight-related behaviours. J Paediatr Child Health $\mathbf{4 9}$, 641-648.

43. Lehto R, Ray C, Lahti-Koski M et al. (2011) Health behaviors, waist circumference and waist-to-height ratio in children. Eur J Clin Nutr 65, 841-848.

44. Howe AS, Mandic S, Parnell WR et al. (2013) Attitudes to food differ between adolescent dieters and non-dieters from Otago, New Zealand, but overall food intake does not. Public Health Nutr 16, 36-45. 